\title{
Article \\ Dairy Product Intake and Long-Term Risk for Frailty among French Elderly Community Dwellers
}

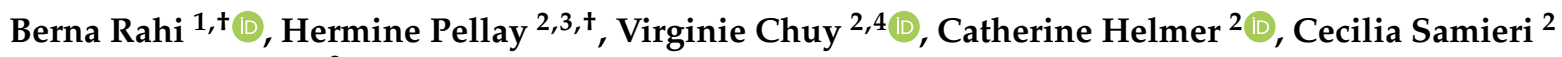 \\ and Catherine Féart ${ }^{2, *}$ \\ 1 Department of Natural Sciences, School of Arts and Sciences, Lebanese American University, \\ Byblos 36, Lebanon; berna.rahi@gmail.com \\ 2 Institut National de la Santé et de la Recherche Médicale (INSERM), University Bordeaux, INSERM, BPH, \\ U1219, F-33000 Bordeaux, France; hermine.pellay@u-bordeaux.fr (H.P.); virginie.chuy@u-bordeaux.fr (V.C.); \\ catherine.helmer@u-bordeaux.fr (C.H.); cecilia.samieri@u-bordeaux.fr (C.S.) \\ 3 CNIEL, Service Recherche Nutrition-Santé, F-75009 Paris, France \\ 4 Department of Dentistry and Oral Health, CHU Bordeaux, University Bordeaux, F-33000 Bordeaux, France \\ * Correspondence: catherine.feart-couret@u-bordeaux.fr \\ + These authors have equally contributed to this work.
}

check for updates

Citation: Rahi, B.; Pellay, H.; Chuy, V.; Helmer, C.; Samieri, C.; Féart, C. Dairy Product Intake and Long-Term Risk for Frailty among French Elderly Community Dwellers. Nutrients 2021, 13, 2151. https://doi.org/10.3390/ nu13072151

Academic Editor: Emmanuel Biver

Received: 5 May 2021

Accepted: 21 June 2021

Published: 23 June 2021

Publisher's Note: MDPI stays neutral with regard to jurisdictional claims in published maps and institutional affiliations.

Copyright: (c) 2021 by the authors. Licensee MDPI, Basel, Switzerland. This article is an open access article distributed under the terms and conditions of the Creative Commons Attribution (CC BY) license (https:// creativecommons.org/licenses/by/ $4.0 /)$.

\begin{abstract}
Dairy products (DP) are part of a food group that may contribute to the prevention of physical frailty. We aimed to investigate DP exposure, including total DP, milk, fresh DP and cheese, and their cross-sectional and prospective associations with physical frailty in community-dwelling older adults. The cross-sectional analysis was carried out on 1490 participants from the Three-City Bordeaux cohort. The 10-year frailty risk was examined in 823 initially non-frail participants. A food frequency questionnaire was used to assess DP exposure. Physical frailty was defined as the presence of at least 3 out of 5 criteria of the frailty phenotype: weight loss, exhaustion, slowness, weakness, and low physical activity. Among others, diet quality and protein intake were considered as confounders. The baseline mean age of participants was $74.1 \mathrm{y}$ and $61 \%$ were females. Frailty prevalence and incidence were $4.2 \%$ and $18.2 \%$, respectively. No significant associations were observed between consumption of total DP or DP sub-types and frailty prevalence or incidence (OR $=1.40,95 \% \mathrm{CI}$ 0.65-3.01 and OR $=1.75,95 \% \mathrm{CI} 0.42-1.32$, for a total DP consumption $>4$ times $/ \mathrm{d}$, respectively). Despite the absence of beneficial associations of higher DP consumption on frailty, older adults are encouraged to follow the national recommendations regarding DP.
\end{abstract}

Keywords: dairy products; frailty; older adults; cohort study

\section{Introduction}

In recent years, the world has been experiencing a steady increase in the aging population. It is expected that by 2050 , one in six people will be over the age of 65 , including one in four in Europe and northern America [1]. This increased life expectancy is associated with a higher risk of morbidities. In fact, nearly a quarter $(23 \%)$ of the overall global burden of death and illness is in people aged over 60, and much of this burden is attributable to longterm illnesses [2]. Advancing age is indeed accompanied by common geriatric syndromes, such as frailty [3]. Frailty is characterized by a depletion in the functional reserves of physiological systems, which limits the possibility to adapt to changes in the environment over time, leading to falls, hospitalization, disability, and death [4]. Nevertheless, frailty can be prevented, and diet appears to be a major determinant of its development $[5,6]$. Several studies have reported that particular macronutrients $[7,8]$, food groups [9-11] and dietary patterns are associated with frailty [12-17]. Particularly, our group has previously reported the relevance of protein intake $(>1 \mathrm{~g} / \mathrm{d}$ being associated with a lower prevalence of frailty) [18], of fruit and vegetable intake ( $>5$ servings/ $d$ being associated with a lower risk of frailty) [9], and of the Mediterranean diet (a higher adherence being associated with 
a lower frailty risk) [17]. In line with our findings, several other longitudinal studies have showed that a higher protein intake is protective against frailty [19-21].

Dietary sources of protein include dairy products (DP), which are also important sources of calcium and vitamin D. Interestingly, recent studies have showed that higher DP consumption was associated with better age-related health outcomes, and particularly lower risks of type 2 diabetes [22,23], cardiovascular diseases and mortality [24,25]. The type of DP (i.e., milk, fresh DP and cheese) appears to be key component of such associations. In fact, a meta-analysis on 938,415 participants and 93,518 mortality cases reported an absence of association between total dairy (high- or low-fat) and milk with the risk of death, while total fermented dairy (including sour milk products, yogurt or cheese; $+20 \mathrm{~g} /$ day) were associated with a significant $2 \%$ reduced risk of all-cause mortality and cardiovascular diseases [26]. While two systematic reviews also observed that higher DP intakes were associated with higher appendicular muscle mass, improved balance-test scores, and an attenuation of the loss of muscle strength $[27,28]$, the direct potential benefit of DP on frailty as a whole has scarcely been studied. To the best of our knowledge, a single prospective study implemented in the Spanish Seniors-ENRICA cohort [29] reported that consuming seven or more servings per week of low-fat milk was associated with a significantly lower risk of frailty compared with consuming less than one serving per week. The external validity of such results remains uncertain. Indeed, the SHARE database demonstrated significant heterogeneity in DP consumption across Europe, with higher levels in central and northern countries and in Spain, and the lowest prevalence of dairy intake in eastern European countries [30]. Of note, high cheese consumption is a hallmark of French dietary habits, and France is also characterized by low milk consumption. Finally, several sociodemographic, nutritional characteristics and lifestyle factors have been associated with the French DP consumption, with specificities according to each DP sub-type [31]. Altogether, it is conceivable that the featured consumption of DP sub-types among French older adults could be differentially associated with frailty.

Therefore, our objective was to assess the cross-sectional and prospective associations between total DP and DP sub-types (milk, fresh DP and cheese) consumption and the 10-year frailty risk among older adults of the Three-City (3C) Bordeaux cohort.

\section{Methods}

\subsection{Study Overview}

The 3C-study is a French population-based prospective study initiated in 1999-2000 to study the vascular risk factors of dementia [32]. Its protocol was approved by the Consultative Committee for the Protection of Persons participating in Biomedical Research at Kremlin-Bicêtre and all participants gave written informed consent. Participants were randomly sampled from electoral rolls from three French cities (Bordeaux, Dijon, and Montpellier). Eligible participants had to be 65 years and older at the time of recruitment and not institutionalized. Among the 9294 participants included at baseline, 2104 were from the Bordeaux center, which completed the initial data collection in 2001-2002 (wave 1). A comprehensive dietary survey of 1597 participants was also performed. This dietary survey served as the baseline for the present study, where DP frequency of consumption and frailty were assessed.

\subsection{Assessment of Dairy Products}

Dietary data were obtained from a semi-quantitative Food Frequency Questionnaire (FFQ) administered during face-to-face interviews by dietitians. This allowed the assessment of the daily frequency of consumption of 148 foods and beverages (with frequencies assessed in 11 classes, from "never or less than once a month" to "7 times per week") during each of the six meals/snacks of the day, as previously detailed [33]. Data from the FFQ was validated against a 24-h dietary recall in an independent subsample of the 3C-study [34]. DP consumption was considered using the frequency of consumption of milk, fresh DP, and cheese. The milk consumption variable included the consumption 
of "milk", "coffee with milk", "tea with milk", "chocolate", "chicory", and "natural milk or with cereal". Consumption of "yogurt and cottage cheese" was classified as fresh DP while frequency of consumption of "cheese" was considered as the cheese category. As already described by Pellay et al. (2020), we considered the DPs' frequency of consumption as four main exposures, including total DP, milk, fresh DP, and cheese [31]. For each DP component, three categories were created based on the quartile distribution of consumption (low frequency: first quartile; intermediate frequency: quartiles 2 and 3; high frequency: fourth quartile). This classification ensured the differentiation between the most infrequent and frequent consumers, as previously described [31].

\subsection{Assessment of Frailty}

At baseline and at the 10-year follow-up, frailty was defined following the Cardiovascular Health Study frailty index [4], the tool recommended by the International Conference of Frailty and Sarcopenia Research [35]. Nevertheless, minor modifications were made to adapt this tool to the available data in our cohort study, as already published [17,18]. Briefly, (1) weight loss was defined as self-reported unintentional loss of $3 \mathrm{~kg}$ or more or, if missing, as a body mass index (BMI) $<21 \mathrm{~kg} / \mathrm{m}^{2}$; (2) exhaustion was evaluated using the following statements from the Center for Epidemiologic Studies-Depression scale (CES-D): "I felt that everything I did was an effort" and "I could not get going". Participants were considered frail for this criterion when they answered "a moderate amount of the time" or "most of the time" to either of these statements [36]; (3) walking speed was determined based on a 6-m walking test, adjusting for height and gender. Participants in the highest quintile were considered slow. When this information was missing, participants were considered frail for this criterion when they reported being unable to walk between $500 \mathrm{~m}$ and $1 \mathrm{~km}$ or to walk up and down a flight of stairs based on the Rosow-Breslau scale [37]. This proxy has been shown to be strongly associated with walking [38]; (4) weakness was identified in different ways at baseline and at the 10-year follow-up, depending on availability of data. At the 10-year follow-up, weakness was identified using the handgrip strength quartiles stratified by sex and BMI, as recommended [4]. At baseline, weakness was identified using the chair standing method, shown to be a good proxy for handgrip strength [39]; (5) physical activity was assessed in a face-to-face interview via an open-ended questionnaire. Low physical activity was defined as less than $1 \mathrm{~h}$ of sports activities or less than $3.5 \mathrm{~h}$ of leisure activities per week, as previously described $[17,18]$.

Older adults with three or more criteria out of five were considered as frail, otherwise they were considered as non-frail. Prevalent frail participants at baseline were excluded for the prospective analyses.

The FRAIL scale was also used to define frailty in sensitivity analyses [40]. The FRAIL scale includes five self-reported components: Fatigue, Resistance, Ambulation, Illnesses and Loss of weight. Fatigue and weight loss were evaluated similarly to those of the frailty index. Resistance and Ambulation were evaluated using the Rosow-Breslau scale, as recommended. Resistance was assessed by asking participants if they could walk up and down a flight of stairs and Ambulation by asking if they could walk between $500 \mathrm{~m}$ and $1 \mathrm{~km}$; "no" responses were each scored as 1 point. Lastly, Illnesses was scored 1 for respondents who reported 5 or more chronic conditions out of 13 including hypertension, diabetes, hypercholesterolemia, cardio- and cerebro-vascular diseases (myocardial infarction or cardiac and vascular surgery, or arteritis or stroke), Parkinson's disease, cognitive decline and dyspnea. Cancer was considered when reports were available, i.e., at the 10 -year follow-up. The FRAIL score ranged from $0-5$, with those scoring three or more considered as frail and those scoring two or less as non-frail.

\subsection{Assessment of Disability}

Dependency in basic Activities of Daily Living (ADLs) was assessed using the five following items of the Katz scale: bathing, dressing, toileting, transferring from bed to chair, and eating [41]. An individual was considered dependent if they could not perform at least 
one activity without a given level of assistance, as defined in the original instrument. All identified dependent participants at baseline and at 10-year follow-up were excluded from the analyses because frailty is considered as risk factor for dependency [35].

\subsection{Covariates}

The covariates included age, sex, marital status, education, smoking status, polypharmacy (dichotomous variable with 6 medications/d as a cut-off), multimorbidity (dichotomous variable with 2 chronic diseases or more as a cut-off point), and global cognitive performances using the Mini-Mental State Examination (MMSE) [42] (0-30 points; higher scores indicate better cognitive status). A diet quality score was also computed. This score included seven components: pulses, raw fruits, raw vegetables, cooked fruits and vegetables, fish, alcohol and olive oil. Each component was dichotomized into meeting the current dietary recommendations versus not. The total score of 7 was also dichotomized into having a good diet quality (score $>3$ ) versus not (score $\leq 3$ ). Finally, total protein intake was evaluated from a single 24-h dietary recall that was administered at home in addition to the FFQ [43].

\subsection{Statistical Analysis}

Baseline demographic, clinical and dietary characteristics were compared between prevalent frail and non-frail (i.e., sample used in the cross-sectional analysis) and incident frail and non-frail (i.e., sample used in the longitudinal analysis) older adults using the student's $t$-test or chi-square test, depending on the type of the variables.

Logistic regression models were used to estimate odds ratio (OR) and $95 \%$ confidence intervals $(95 \% \mathrm{CI})$ for the association between consumption of total DP or DP sub-type (milk, fresh DP, or cheese) and frailty, both cross-sectionally and prospectively. For each DP exposure, intermediate frequency consumption (quartiles 2 and 3) and high frequency consumption (quartile 4) were compared to the reference category of low frequency consumption (quartile 1).

Model 1 was adjusted for age, sex, education and marital status. Model 2 was additionally adjusted for smoking status, multimorbidity, polypharmacy, diet quality score, total protein intake and global cognitive performances. Finally, two sets of sensitivity analyses were performed. First, we assessed frailty using the FRAIL scale and the same multivariate models were applied, except excluding multimorbidity as a covariate from model 2 as this variable is a component of the FRAIL scale. Second, we retained all ADL dependent individuals as we assumed that those who are ADL dependent might already be frail, both in the cross-sectional analysis and the prospective one. All statistical analyses were performed with the SAS Statistical package (Version 9.4 SAS Institute) and statistical significance was set at $p<0.05$.

\section{Results}

\subsection{Sample Characteristics}

Among 1597 participants who answered the dietary survey at baseline, 107 were excluded from all analyses for the following reasons: 20 were ADL dependent at baseline, 67 could not be classified for frailty, 9 had missing information about DP consumption and 11 participants had missing information for covariates. Therefore, the final sample for the cross-sectional analysis comprised 1490 participants (including 1427 non-frail). Among those participants, 979 (69\%) were followed up at 10 years (during the follow-up, 355 participants died). An additional 156 participants were excluded from longitudinal analyses $(n=79$ participants were identified as dependent and $n=77$ with missing frailty status at 10 years). Thus, 823 participants were prospectively analyzed (Figure 1). 


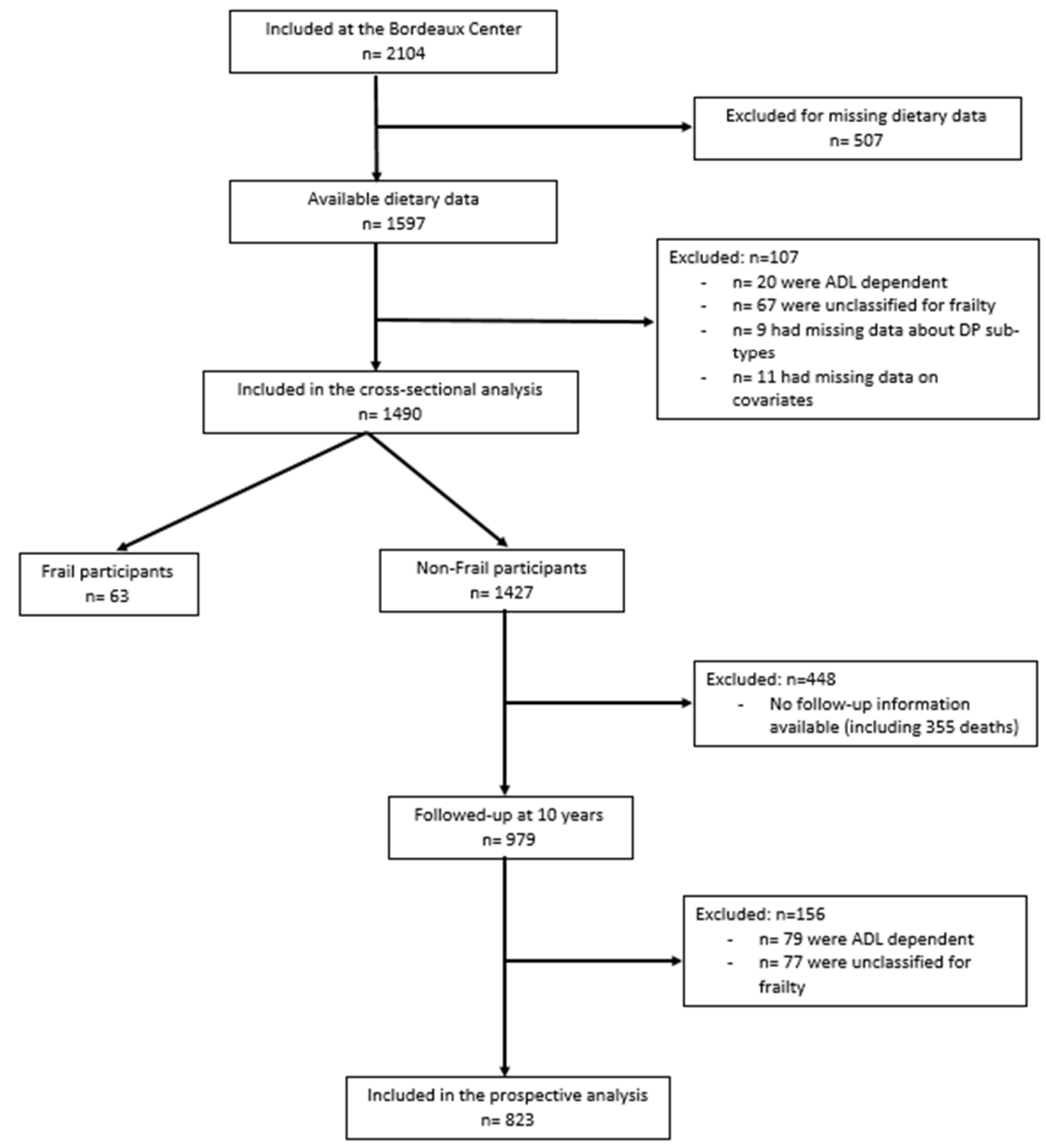

Figure 1. Flow chart of the cross-sectional and the prospective studied samples. Three-City Bordeaux Study, 2000-2010.

In cross-sectional analyses, the studied sample $(n=1490)$ constituted mainly of females $(n=906,60.8 \%)$ and had an average age of $74.1 \pm 4.9$ (standard deviation) years (Table 1$)$. Half the sample was married (57\%) and reported multimorbidity $(48 \%)$, and a third (32\%) was taking 6 medications / $d$ or more. Prevalence of frailty was $4.2 \%(n=63)$. The most prevalent frailty criterion was low physical activity $(n=234,20.1 \%)$ followed by slow walking speed ( $n=281,19 \%$ ) while the least prevalent frailty criterion was muscle weakness $(n=77,5.3 \%)$ followed by weight loss $(n=82,5.5 \%)$. Those included in prospective analyses $(n=823)$ were non-frail participants at baseline, mainly females $(65.0 \%)$ and were on average $72.8 \pm 4.4$ years old. A total of 150 participants $(18.2 \%)$ exhibited frailty at the 10 -year follow-up and the most incident frailty criterion was low physical activity $(n=473$, $58.2 \%)$ followed by muscle weakness $(n=199,26.6 \%)$. The least incident frailty criterion was weight loss $(n=69,8.4 \%)$ followed by exhaustion $(n=138,17.6 \%)$. 
Table 1. Socio-demographic, clinical, dietary, and lifestyle characteristics according to the frailty prevalence (cross-sectional sample, $n=1490$ in 2000) and incidence (prospective sample, $n=823$ between 2000-2010) of older adults from the Three-City study, Bordeaux (France).

\begin{tabular}{|c|c|c|c|c|c|c|c|c|}
\hline & \multicolumn{4}{|c|}{ Cross-Sectional Analyses } & \multicolumn{4}{|c|}{ Prospective Analyses } \\
\hline & $\begin{array}{c}\text { Overall } \\
(n=1490)\end{array}$ & $\begin{array}{c}\text { Frail at } \\
\text { Baseline } \\
(n=63)\end{array}$ & $\begin{array}{c}\text { Non-Frail at } \\
\text { Baseline } \\
(n=1427)\end{array}$ & $p^{\mathrm{a}}$ & $\begin{array}{c}\text { Overall } \\
(n=823)\end{array}$ & $\begin{array}{l}\text { Non-Frail at } \\
\text { Follow-Up } \\
(n=673)\end{array}$ & $\begin{array}{c}\text { Incident } \\
\text { Frail } \\
(n=150)\end{array}$ & $p^{a}$ \\
\hline \multicolumn{9}{|c|}{ Demographic characteristics $(n, \%) *$} \\
\hline Sex (females) & $906(60.81)$ & $43(68.25)$ & $863(60.48)$ & 0.22 & $535(65.01)$ & $433(64.34)$ & $102(68.00)$ & 0.40 \\
\hline Age (y), mean (SD) & $74.10(4.90)$ & $77.69(5.24)$ & $73.95(4.82)$ & $<0.0001$ & $72.80(4.35)$ & $72.20(4.08)$ & $75.49(4.50)$ & $<0.0001$ \\
\hline Education & & & & 0.27 & & & & 0.11 \\
\hline No/Primary & $478(32.10)$ & $26(41.27)$ & $452(31.67)$ & & $232(28.19)$ & $180(26.75)$ & $52(34.67)$ & \\
\hline Secondary or high & $731(49.10)$ & $26(41.27)$ & $705(49.40)$ & & $424(51.52)$ & 357 (53.05) & $67(44.67)$ & \\
\hline University & $281(18.90)$ & $11(17.46)$ & $270(18.92)$ & & $167(20.29)$ & $136(20.21)$ & $31(20.67)$ & \\
\hline Marital Status & & & & 0.053 & & & & 0.001 \\
\hline Married & $849(57.00)$ & $26(41.27)$ & $823(57.67)$ & & $471(57.23)$ & $402(59.73)$ & $69(46.00)$ & \\
\hline Divorced/separated & $118(7.90)$ & $5(7.94)$ & $113(7.92)$ & & $64(7.78)$ & $56(8.32)$ & $8(5.33)$ & \\
\hline Widowed & $429(28.80)$ & $27(42.86)$ & $402(28.17)$ & & $228(27.70)$ & $168(24.96)$ & $60(40.00)$ & \\
\hline Single & $94(6.30)$ & $5(7.94)$ & $89(6.24)$ & & $60(7.29)$ & $47(6.98)$ & $13(8.67)$ & \\
\hline \multicolumn{9}{|c|}{ Clinical characteristics $(n, \%) *$} \\
\hline Smoking status & & & & 0.45 & & & & 0.54 \\
\hline Never smoker & $937(62.90)$ & $44(69.84)$ & $893(62.58)$ & & $540(65.61)$ & $442(65.68)$ & $98(65.33)$ & \\
\hline Ex-smoker & $472(31.70)$ & $17(26.98)$ & 455 (31.89) & & $242(29.40)$ & 195 (28.97) & 47 (31.33) & \\
\hline Current & $81(5.40)$ & $2(3.17)$ & $79(5.54)$ & & $41(4.98)$ & $36(5.35)$ & $5(3.33)$ & \\
\hline $\begin{array}{l}\text { MMSE score }{ }^{b} \text {, mean } \\
\text { (SD) }\end{array}$ & $27.52(1.98)$ & $26.43(2.34)$ & 27.57 (1.96) & $<0.0001$ & $27.84(1.79)$ & 27.89 (1.76) & $27.61(1.90)$ & 0.08 \\
\hline Medications $/ \mathrm{d} \geq 6$ & $480(32.20)$ & $38(60.31)$ & $442(30.97)$ & $<0.0001$ & $220(26.73)$ & $155(23.03)$ & $65(43.33)$ & $<0.0001$ \\
\hline Multimorbidity & $727(48.8)$ & $41(65.08)$ & $686(48.07)$ & 0.008 & $395(48.00)$ & $299(44.43)$ & $96(64.00)$ & $<0.0001$ \\
\hline \multicolumn{9}{|c|}{ Nutritional and dietary characteristics } \\
\hline $\begin{array}{l}\mathrm{BMI}\left(\mathrm{kg} / \mathrm{m}^{2}\right) \\
\quad(\mathrm{m}=18)\end{array}$ & $26.33(4.16)$ & $27.51(6.10)$ & $26.28(4.06)$ & 0.03 & $26.25(3.95)$ & $26.15(3.83)$ & $26.68(4.43)$ & 0.15 \\
\hline BMI categories & & & & 0.16 & & & & 0.63 \\
\hline$<23$ & $307(20.86)$ & $12(21.05)$ & $295(20.85)$ & & $172(21.00)$ & $142(21.19)$ & $30(20.13)$ & \\
\hline $23-27$ & $577(39.20)$ & $16(28.07)$ & $561(39.65)$ & & $329(40.17)$ & $273(40.75)$ & $56(37.58)$ & \\
\hline$>27$ & $588(39.95)$ & $29(50.88)$ & $559(39.51)$ & & $318(38.83)$ & 255 (38.06) & $63(42.28)$ & \\
\hline Total protein $(\mathrm{g} / \mathrm{d})$ & $75.75(26.80)$ & $72.14(27.05)$ & $75.91(26.79)$ & 0.27 & $75.36(26.22)$ & $76.05(25.98)$ & $72.23(27.12)$ & 0.11 \\
\hline $\begin{array}{c}\text { Diet index }(n, \% \text { high } \\
\text { quality) }\end{array}$ & $761(51.1)$ & $32(50.79)$ & 729 (51.09) & 0.96 & $423(51.40)$ & 357 (53.05) & $66(44.00)$ & 0.05 \\
\hline \multicolumn{9}{|c|}{ Items of the phenotype of frailty } \\
\hline Weight loss & $\begin{array}{l}82(5.51) \\
(\mathrm{m}=1)\end{array}$ & $27(43.55)$ & $55(3.85)$ & $<0.0001$ & $\begin{array}{c}69(8.42) \\
\mathrm{m}=4\end{array}$ & $35(5.22)$ & $34(22.97)$ & $<0.0001$ \\
\hline Exhaustion & $\begin{array}{l}223(15.12) \\
(\mathrm{m}=15)\end{array}$ & $53(84.13)$ & $170(12.04)$ & $<0.0001$ & $\begin{array}{c}138(17.56) \\
\mathrm{m}=37\end{array}$ & $54(8.32)$ & $84(61.31)$ & $<0.0001$ \\
\hline Muscle weakness & $\begin{array}{l}77(5.25) \\
(\mathrm{m}=24)\end{array}$ & $35(58.33)$ & $42(2.99)$ & $<0.0001$ & $\begin{array}{c}199(26.57) \\
\mathrm{m}=74\end{array}$ & $107(17.31)$ & $92(70.23)$ & $<0.0001$ \\
\hline Walking speed & $\begin{array}{l}281(18.90) \\
\quad(\mathrm{m}=3)\end{array}$ & $53(84.12)$ & $228(16.01)$ & $<0.0001$ & $\begin{array}{l}209(25.49) \\
\mathrm{m}=3\end{array}$ & $81(12.09)$ & $128(85.33)$ & $<0.0001$ \\
\hline Physical activity & $\begin{array}{l}234(20.05) \\
(\mathrm{m}=323)\end{array}$ & $36(78.26)$ & 198 (17.66) & $<0.0001$ & $\begin{array}{c}473(58.18) \\
\mathrm{m}=10\end{array}$ & 329 (49.47) & 144 (97.30) & $<0.0001$ \\
\hline
\end{tabular}

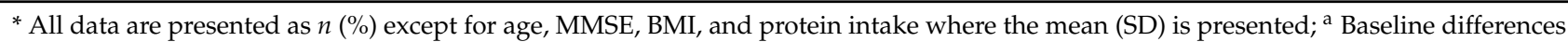
between prevalent frail and non-frail $(n=1490)$ and incident frail and non-frail $(n=823)$ participants tested by $t$-tests or chi square tests depending on the type of the variable; ${ }^{\mathrm{b}}$ Mini-Mental State Examination; $\mathrm{m}=$ missing.

Prevalent and incident frail older adults were significantly older, were more likely to be depressed, to take 6 medications/day or more, and to have comorbidities at baseline compared with prevalent non-frail participants, and with participants free from frailty over time, respectively (Table 1). Moreover, prevalent frail participants exhibited a significantly higher BMI on average than non-frail participants, and the daily consumption of proteins was not significantly different between the frail and non-frail participants at baseline (i.e., cross-sectional sample). Regarding the sample enrolled in prospective analyses, incident frail participants had a similar BMI compared to those who remained free from frailty, while a higher percentage of incident frail participants had a lower diet quality score compared with participants who remained free from frailty ( $53 \%$ vs. $44 \%, p=0.045$ ). 
Frequencies of consumption of DP (total DP, milk, fresh DP, and cheese) are presented in Table 2 for both cross-sectional and prospective samples. No significant differences were observed between prevalent frail and non-frail or incident frail and non-frail participants regarding the frequency of total DP and DP-subtypes consumption at baseline.

Table 2. Frequency of consumption of dairy products (total and sub-types) according to the frailty prevalence (cross-sectional sample, $n=1490$ in 2000) and incidence (prospective sample, $n=823$ between 2000-2010) of older adults from the Three-City study, Bordeaux (France).

\begin{tabular}{|c|c|c|c|c|c|c|c|c|}
\hline & \multicolumn{4}{|c|}{ Cross-Sectional Analyses } & \multicolumn{4}{|c|}{ Prospective Analyses } \\
\hline & $\begin{array}{c}\text { Overall } \\
(n=1490)\end{array}$ & $\begin{array}{c}\text { Frail at } \\
\text { Baseline } \\
(n=63)\end{array}$ & $\begin{array}{c}\text { Non-Frail at } \\
\text { Baseline } \\
(n=1427)\end{array}$ & $p^{\mathrm{a}}$ & $\begin{array}{c}\text { Overall } \\
(n=823)\end{array}$ & $\begin{array}{l}\text { Non-Frail at } \\
\text { Follow-Up } \\
(n=673)\end{array}$ & $\begin{array}{c}\text { Incident } \\
\text { Frail } \\
(n=150)\end{array}$ & $p^{\mathrm{a}}$ \\
\hline Total Dairy Products & & & & 0.37 & & & & 0.58 \\
\hline Low: $\leq 2$ times $/ \mathrm{d}$ & $375(25.17)$ & $13(20.63)$ & $362(25.37)$ & & $198(24.06)$ & $157(23.33)$ & $41(27.33)$ & \\
\hline Intermediate: $2-4$ times $/ \mathrm{d}$ & $769(51.61)$ & $31(49.21)$ & $738(51.72)$ & & $439(53.34)$ & $362(53.79)$ & $77(51.33)$ & \\
\hline High: $\geq 4$ times $/ \mathrm{d}$ & $346(23.22)$ & $19(30.16)$ & $327(22.92)$ & & $186(22.60)$ & $154(22.88)$ & $32(21.33)$ & \\
\hline Milk & & & & 0.37 & & & & 0.54 \\
\hline Low: 0 times $/ \mathrm{d}$ & $433(29.06)$ & $17(26.98)$ & $416(29.15)$ & & $229(27.83)$ & $182(27.04)$ & $47(31.33)$ & \\
\hline Intermediate: $0-1$ times $/ \mathrm{d}$ & $716(48.05)$ & $27(42.86)$ & $689(48.28)$ & & $383(46.54)$ & $318(47.25)$ & $65(43.33)$ & \\
\hline High: $>1$ times $/ \mathrm{d}$ & $341(22.89)$ & $19(30.16)$ & $322(22.56)$ & & $211(25.64)$ & $173(25.71)$ & $38(25.33)$ & \\
\hline Fresh dairy products & & & & 0.52 & & & & 0.46 \\
\hline Low: $<0.5$ times $/ \mathrm{d}$ & $408(27.38)$ & $16(25.40)$ & $392(27.47)$ & & $207(25.15)$ & $167(24.81)$ & $40(26.67)$ & \\
\hline Intermediate: $0.5-1.5$ times $/ \mathrm{d}$ & $723(48.52)$ & $28(44.44)$ & $695(48.70)$ & & $419(50.91)$ & $339(50.37)$ & $80(53.33)$ & \\
\hline High: $>1.5$ times $/ \mathrm{d}$ & $359(24.09)$ & $19(30.16)$ & $340(23.83)$ & & $197(23.94)$ & $167(24.81)$ & $30(20.00)$ & \\
\hline Cheese & & & & 0.39 & & & & 0.31 \\
\hline Low: $\leq 0.5$ times $/ \mathrm{d}$ & 297 (19.93) & $16(19.69)$ & $281(19.69)$ & & $174(21.14)$ & $143(21.25)$ & $31(20.67)$ & \\
\hline Intermediate: $0.5-1.5$ times $/ \mathrm{d}$ & $779(52.28)$ & $28(52.63)$ & $751(52.63)$ & & $477(54.31)$ & $372(55.27)$ & $75(50.00)$ & \\
\hline High: $>1.5$ times $/ \mathrm{d}$ & $414(27.79)$ & $19(30.16)$ & $395(27.68)$ & & $202(24.54)$ & $158(23.48)$ & $44(29.33)$ & \\
\hline
\end{tabular}

All data are presented as $n(\%) ;{ }^{\text {a }}$ Baseline differences between prevalent frail and non-frail $(n=1490)$ and incident frail and non-frail $(n=823)$ participants tested by $t$-tests or chi square tests depending on the type of the variable; $\mathrm{m}=$ missing.

\subsection{Associations between Spectrum of DP Exposure and Prevalence of Frailty}

In models adjusted for age, sex, marital status and education, we did not observe any significant association between total DP and DP sub-types and frailty prevalence, when comparing the highest frequency to the lowest frequency consumption of DP (Table 3). In models additionally adjusted for smoking status, multimorbidity, polypharmacy, protein intake, diet quality and global cognitive score, all associations with the prevalence of frailty remained not significant for all DP exposures: total DP $(\mathrm{OR}=1.08,95 \% \mathrm{CI}=0.54-2.17$ and $1.40,95 \% \mathrm{CI}=0.65-3.01$ for intermediate and high consumption vs. low, respectively), milk $(\mathrm{OR}=1.13,95 \% \mathrm{CI}=0.56-2.31)$, fresh $\mathrm{DP}(\mathrm{OR}=1.13,95 \% \mathrm{CI}=0.54-2.33)$, and cheese $(\mathrm{OR}=0.89 ; 95 \% \mathrm{CI}=0.43-1.88)$ for high vs. low frequency of consumption.

\subsection{Associations between Spectrum of DP Exposure and Incidence of Frailty}

When focusing on the 10-year risk for frailty, we observed that baseline frequencies of consumption of total DP and DP sub-types were not significantly associated with the frailty risk when we compared the lowest frequency to the highest frequency of consumption of total DP (OR $=0.74,95 \% \mathrm{CI}=0.42-1.30)$, milk (OR $=0.80,95 \% \mathrm{CI}=0.48-1.35)$, fresh DP $(\mathrm{OR}=0.68,95 \% \mathrm{CI}=0.38-1.20)$ and cheese consumption $(\mathrm{OR}=1.19,05 \% \mathrm{CI}=0.68-2.10)$ in fully adjusted models (Table 4).

\subsection{Sensitivity Analyses}

The FRAIL scale was also implemented to alternatively identify prevalent and incident frail participants. Sixty out of 1552 participants (3.9\%) were considered as frail at baseline according to this scale. In fully adjusted models (i.e., model 2 ), all associations with the prevalence of frailty were not significant for all DP exposures: total DP $(\mathrm{OR}=1.42,95 \% \mathrm{CI}=0.64-3.13)$, milk $(\mathrm{OR}=1.11,95 \% \mathrm{CI}=0.54-2.32)$, fresh DP $(\mathrm{OR}=0.96$, $95 \% \mathrm{CI}=0.46-1.98)$, and cheese $(\mathrm{OR}=0.86 ; 95 \% \mathrm{CI}=0.39-1.88)$ for high vs. low frequency of consumption. Among 1492 non-frail non-dependent, 1006 were followed at 
the 10-year follow-up (lost to follow-up $n=486$ ). Among those, an additional 87 were excluded from the analysis because they were ADL dependent and another 23 were excluded because they were unclassified for the FRAIL scale, leading to a final sample size of 896 , with $45(5.0 \%)$ classified as frail on the FRAIL scale. Regarding the spectrum of DP exposures, we only observed that the highest, compared with the lowest, frequency of consumption of fresh DP was associated with lower frailty risk in the fully adjusted model $(\mathrm{OR}=0.35,95 \% \mathrm{CI}=0.13-0.97, p=0.04, p$ global $=0.13)$ while all other associations were non-significant, for instance, total $\mathrm{DP}(\mathrm{OR}=1.66,95 \% \mathrm{CI}=0.26-1.67)$, milk (OR=1.21, $95 \% \mathrm{CI}=0.49-2.98)$, and cheese $(\mathrm{OR}=1.25 ; 95 \% \mathrm{CI}=0.49-3.21)$ for high vs. low frequency of consumption.

Second, when all ADL dependent individuals were maintained in analytic samples, 1501 and 885 participants were included for the cross-sectional and prospective analyses, respectively. Among those 1501 participants, 68 (4.5\%) were identified as frail. None of the total DP or DP sub-types exposures were associated with frailty prevalence in the fully adjusted models: total $\mathrm{DP}(\mathrm{OR}=1.44,95 \% \mathrm{CI}=0.68-3.04)$, milk $(\mathrm{OR}=1.04,95 \% \mathrm{CI}=0.52-2.1)$, fresh DP (OR $=1.21,95 \% \mathrm{CI}=0.59-2.48)$, and cheese $(\mathrm{OR}=0.85 ; 95 \% \mathrm{CI}=0.42-1.74)$ for high vs. low frequency of consumption. Among those 1433 non-frail at baseline, 449 were lost at the 10-year follow-up and 99 were unclassified for frailty incidence, leading to a final sample size of 885 for the prospective analyses. At the 10-year follow-up, 195 participants $(22 \%)$ were identified as frail. Regarding the spectrum of DP exposures, none of the frequency of consumption of total DP or DP sub-types were significantly associated with frailty risk in the fully adjusted models: total DP $(\mathrm{OR}=0.65,95 \% \mathrm{CI}=0.39-1.09)$, milk ( $\mathrm{OR}=0.61,95 \% \mathrm{CI}=0.38-1.00)$, fresh DP $(\mathrm{OR}=0.64,95 \% \mathrm{CI}=0.39-1.08)$, and cheese $(\mathrm{OR}=1.4 ; 95 \% \mathrm{CI}=0.83-2.41)$ for high vs. low frequency of consumption.

Table 3. Multivariate association between baseline frequencies of consumption of total DP, milk, fresh DP, and cheese and frailty prevalence among older adults in the Three-City Study, Bordeaux $(n=1490,2000)$.

\begin{tabular}{|c|c|c|c|c|c|}
\hline \multicolumn{6}{|c|}{ Total Dairy Products Frequency of Consumption } \\
\hline & $n$ frail/Total & Model 1 & & Model 2 & \\
\hline & & OR $(95 \%$ CI) & $p$ & OR $(95 \% \mathrm{CI})$ & $p$ \\
\hline Low: $\leq 2$ times $/ \mathrm{d}$ & $13 / 375$ & Ref & & Ref & \\
\hline Intermediate: $2-4$ times $/ \mathrm{d}$ & $31 / 769$ & $1.07(0.54-2.09)$ & 0.84 & $1.08(0.54-2.17)$ & 0.82 \\
\hline High: $\geq 4$ times $/ \mathrm{d}$ & $19 / 346$ & $1.50(0.72-3.13)$ & 0.28 & $1.40(0.65-3.01)$ & 0.39 \\
\hline Global p & & & 0.45 & & 0.62 \\
\hline \multicolumn{6}{|c|}{ Milk Frequency of Consumption } \\
\hline & $n$ frail/Total & Model 1 & & Model 2 & \\
\hline & & OR $(95 \% \mathrm{CI})$ & $p$ & OR $(95 \% \mathrm{CI})$ & $p$ \\
\hline Low: 0 times $/ \mathrm{d}$ & $17 / 433$ & Ref & & Ref & \\
\hline Intermediate: $0-1$ times/d & $27 / 716$ & $0.91(0.49-1.71)$ & 0.77 & $0.83(0.44-1.58)$ & 0.57 \\
\hline High: $>1$ times $/ \mathrm{d}$ & $19 / 341$ & $1.21(0.61-2.41)$ & 0.58 & $1.13(0.56-2.31)$ & 0.73 \\
\hline Global p & & & 0.66 & & 0.62 \\
\hline \multicolumn{6}{|c|}{ Fresh Dairy Products Frequency of Consumption } \\
\hline & $n$ frail/Total & Model 1 & & Model 2 & \\
\hline & & OR $(95 \% \mathrm{CI})$ & $p$ & OR $(95 \% \mathrm{CI})$ & $p$ \\
\hline Low: $<0.5$ times $/ \mathrm{d}$ & $16 / 408$ & Ref & & Ref & \\
\hline Intermediate: $0.5-1.5$ times $/ \mathrm{d}$ & $28 / 723$ & $0.90(0.47-1.72)$ & 0.76 & $0.83(0.43-1.62)$ & 0.57 \\
\hline High: $>1.5$ times $/ \mathrm{d}$ & $19 / 359$ & $1.22(0.60-2.48)$ & 0.58 & $1.13(0.54-2.33)$ & 0.74 \\
\hline Global p & & & 0.62 & & 0.62 \\
\hline \multicolumn{6}{|c|}{ Cheese Frequency of Consumption } \\
\hline & $n$ frail/Total & Model 1 & & Model 2 & \\
\hline & & OR $(95 \% \mathrm{CI})$ & $p$ & OR $(95 \% \mathrm{CI})$ & $p$ \\
\hline Low: $\leq 0.5$ times $/ \mathrm{d}$ & $16 / 297$ & Ref & & Ref & \\
\hline Intermediate: $0.5-1.5$ times $/ \mathrm{d}$ & $28 / 779$ & $0.66(0.35-1.27)$ & 0.22 & $0.68(0.38-1.32)$ & 0.25 \\
\hline High: $>1.5$ times $/ \mathrm{d}$ & $19 / 414$ & $0.92(0.45-1.86)$ & 0.81 & $0.89(0.43-1.88)$ & 0.77 \\
\hline Global p & & & 0.38 & & 0.45 \\
\hline
\end{tabular}

OR: Odds ratio, CI: Confidence Intervals; Model 1: Model adjusted for age, sex, marital status and education; Model 2: Model 1 + additional adjustment for smoking status, multimorbidity, polypharmacy, total protein, diet quality score, and Mini-Mental State Examination. 
Table 4. Multivariate association between baseline frequencies of consumption of total DP, milk, fresh DP, and cheese and the 10-year frailty risk among older adults in the Three-City Study, Bordeaux $(n=823,2000-2010)$.

\begin{tabular}{|c|c|c|c|c|c|}
\hline \multicolumn{6}{|c|}{ Total Dairy Products Frequency of Consumption } \\
\hline & \multirow[t]{2}{*}{$n$ frail/Total } & \multirow{2}{*}{$\begin{array}{c}\text { Model } 1 \\
\text { OR }(95 \% \text { CI) }\end{array}$} & \multicolumn{3}{|c|}{ Model 2} \\
\hline & & & $p$ & OR $(95 \% \mathrm{CI})$ & $p$ \\
\hline Low: $\leq 2$ times $/ \mathrm{d}$ & $41 / 198$ & Ref & & Ref & \\
\hline Intermediate: $2-4$ times $/ \mathrm{d}$ & $77 / 439$ & $0.70(0.44-1.09)$ & 0.11 & $0.73(0.46-1.16)$ & 0.19 \\
\hline High: $\geq 4$ times $/ \mathrm{d}$ & $32 / 186$ & $0.76(0.44-1.30)$ & 0.19 & $0.75(0.42-1.32)$ & 0.32 \\
\hline \multirow[t]{4}{*}{ Global p } & & & 0.28 & & 0.40 \\
\hline & \multicolumn{3}{|c|}{ Milk Frequency of Consumption } & & \\
\hline & \multirow[t]{2}{*}{$n$ frail/Total } & Model 1 & \multicolumn{3}{|c|}{ Model 2} \\
\hline & & OR $(95 \% \mathrm{CI})$ & $p$ & OR $(95 \% \mathrm{CI})$ & $p$ \\
\hline Low: 0 times $/ \mathrm{d}$ & $47 / 229$ & Ref & & Ref & \\
\hline Intermediate: $0-1$ times $/ \mathrm{d}$ & $65 / 383$ & $0.47(0.50-1.21)$ & 0.26 & $0.79(0.50-1.22)$ & 0.28 \\
\hline High: $>1$ times/d & $38 / 211$ & $0.75(0.45-1.24)$ & 0.26 & $0.78(0.46-1.31)$ & 0.34 \\
\hline \multirow[t]{4}{*}{ Global p } & \multirow{2}{*}{\multicolumn{4}{|c|}{ Fresh Dairy Products Frequency of Consumption }} & 0.50 \\
\hline & & & & & \\
\hline & \multirow[t]{2}{*}{$n$ frail/Total } & Model 1 & \multicolumn{3}{|c|}{ Model 2} \\
\hline & & OR $(95 \%$ CI $)$ & $p$ & OR $(95 \%$ CI $)$ & $p$ \\
\hline Low: $<0.5$ times $/ \mathrm{d}$ & $40 / 207$ & Ref & & Ref & \\
\hline Intermediate: $0.5-1.5$ times $/ \mathrm{d}$ & $80 / 419$ & $0.92(0.58-1.44)$ & 0.71 & $0.91(0.57-1.45)$ & 0.68 \\
\hline High: $>1.5$ times $/ \mathrm{d}$ & $30 / 197$ & $0.66(0.38-1.16)$ & 0.15 & $0.65(0.57-1.16)$ & 0.15 \\
\hline \multirow[t]{4}{*}{ Global p } & & & 0.31 & & 0.31 \\
\hline & \multicolumn{3}{|c|}{ Cheese Frequency of Consumption } & & \\
\hline & $n$ frail/Total & Model 1 & & Model 2 & \\
\hline & & OR $(95 \% \mathrm{CI})$ & $p$ & OR $(95 \% \mathrm{CI})$ & $p$ \\
\hline Low: $\leq 0.5$ times $/ \mathrm{d}$ & $31 / 174$ & Ref & & Ref & \\
\hline Intermediate: $0.5-1.5$ times $/ \mathrm{d}$ & $75 / 447$ & $0.86(0.53-1.40)$ & 0.54 & $0.86(0.52-1.41)$ & 0.54 \\
\hline High: $>1.5$ times $/ \mathrm{d}$ & $44 / 202$ & $1.31(0.76-2.27)$ & 0.33 & $1.25(0.71-2.21)$ & 0.44 \\
\hline Global p & & & 0.17 & & 0.27 \\
\hline
\end{tabular}

OR: Odds ratio, CI: Confidence Intervals; Model 1: Model adjusted for age, sex, marital status and education; Model 2: Model $1+$ additional adjustment for smoking status, multimorbidity, polypharmacy, total protein, diet quality score, and Mini-Mental State Examination.

\section{Discussion}

In the present analysis of French community-dwelling older adults enrolled in the 3C-Bordeaux study, the frequency of DP consumption was not significantly associated with frailty, assessed using proxies of the frailty phenotype, in either cross-sectional or prospective analyses. In particular, total DP, milk, fresh DP and cheese were not associated with frailty prevalence at baseline. Similarly, these food groups were not associated with frailty risk at 10 years. Similar results were observed when frailty was assessed using the FRAIL scale, strengthening our conclusions.

Several studies have evaluated the association between DP and age-related chronic diseases and mortality $[22,23,44]$. Nevertheless, to our knowledge, very few studies have evaluated the relationship between DP and frailty and their results were mixed. A crosssectional study evaluated the association between dairy intake and physical function among1456 older women aged 70 to 85 years [45]. The authors observed that compared to those in the lowest tertile of dairy consumption, those in the highest tertile of consumption had significantly higher handgrip strength and lower odds for a poor Timed Up and Go while no differences were observed in the prevalence of falls. In a sample of 1871 Spanish older adults enrolled in the Seniors-ENRICA cohort [29], greater consumption of low-fat dairy products and low-fat milk in particular was associated with lower frailty risk over 3.5 years of follow-up, while no significant results were observed for whole milk, yogurt, cheese and low-fat yogurt. Contrary to the presented studies, our findings did not show any association with frailty prevalence or risk at the 10-year follow-up. Interestingly, our 
results are similar to a recent analysis of the InCHIANTI study where the main objective was to evaluate the associations between adherence to a Mediterranean-type diet (MeDi) and frailty index at baseline and at the 10-year follow-up [46]. In a sub-analysis, the authors investigated the effect of individual components of the MeDi and frailty, and they observed that DP intake was not significantly associated with frailty in both the cross-sectional and prospective analyses.

Several possible explanations could justify the absence of associations between DP and frailty in our sample. First, the FFQ used to collect dietary data assessed the frequency of consumption only, while information about the quantities, which could have been interesting, were only assessed by a single 24-h dietary recall (which questions its relevance). Therefore, despite the higher consumption frequency, this intake might have been below what is recommended and therefore affected our results. In fact, in a recent analysis of the 3C-study participants describing their DP intake at baseline, it was observed that participants with the highest frequency of total DP per day consumed lower than the recommended intakes [31]. These results were in line with a previous national report where it was observed that $64 \%$ of participants aged 55 to 79 years old reported consumption below recommendations [47]. Second, in the 3C-study, total DP consumption and its sub-types have been previously shown to be associated with different eating patterns [31]. Although we have adjusted for diet quality in our analyses, we cannot exclude the possibility of some residual confounding that has led to an absence of significance. This is noteworthy as it was observed that higher total DP consumption was associated with a higher consumption of biscuits, sweets and cooked vegetables, and higher frequency of milk consumption was significantly associated with higher intakes of biscuit and sweets, a dietary pattern described as "biscuits and snacking" in the 3C-study. Moreover, it was observed that the highest frequency consumers of fresh DP had a low total energy intake. Finally, the highest frequency of cheese consumption was associated with a high consumption of cereals and grains, sweets, charcuterie, meat, poultry, and alcohol [31]. These results showed that the higher consumption of total DP or sub-types was associated with less-than-optimal diets, rich in sugar and saturated fatty acids, part of a western-type diet [48] and potential risk factors for frailty $[14,49,50]$. For instance, in a cross-sectional NHANES study including 4062 participants $\geq 50$ y of age, a higher percentage of saturated fatty acid intake was associated with higher frailtyprevalence [50]. Therefore, we speculated that the null association between highest DP consumption and frailty observed here might be the result of possible positive effects of some favorable nutrients on frailty (i.e., higher protein and energy intake), but attenuated by the possible negative effects of saturated fatty acid intake and the overall diet quality, although we have controlled for components of the diet in the analyses. Third, no information was available about the quality of DP, whether they were natural or sweetened, or fermented or not. In fact, flavored milk, whole yogurt and fermented milk, dairy desserts and sweetened cheeses are all sources of added sugars, which were shown to be associated with an increased risk of frailty in an analysis of the Seniors ENRICA cohort [49]. The highest tertile of added sugars consumption was associated with a higher frailty risk (i.e., multiplied by 2.3) compared to those in the lowest tertile. Finally, unlike the analyses from the Seniors-ENRICA cohort study [29], we were not able to differentiate between types of DP consumed based on their fat content. Nevertheless, the 24-h dietary recall administered at baseline of the 3C-Bordeaux study showed that only $7 \%$ of the participants had whole-fat milk and among those, only $10 \%$ reported regular consumption of whole-fat milk while up to $25 \%$ of the sample consumed whole fresh DP and 19\% consumed flavored fresh DP or yogurt with fruits (unpublished data). This might imply that factors other than the fat content of DP might play a role in the association between DP and frailty. Altogether, we speculated that the observed null association between highest DP consumption (whatever the subtypes) and frailty might be the results of interactions between the different concentrations of beneficial and harmful ingredients leading to an unbalanced quality of DP and of related dietary patterns. 
The present study has some methodological limitations. First, as previously stated, we had no detailed information about the portion sizes and this would have affected our results as national recommendations emphasize the quantity consumed rather than frequency. Moreover, a high frequency consumption does not necessarily mean reaching the recommended levels, as older adults might have frequent but smaller intakes. Therefore, the inability to evaluate the portion sizes might have hindered any potential association of DP with frailty status. We also did not assess DP intake from mixed dishes, and this might have led to underestimation of the DP consumption frequency. This is an important issue to consider in future studies as milk is a recurrent constituent of several French recipes. Another limitation is that we did not adjust for important micronutrients related to DP and associated with frailty, namely, vitamin D [51,52]. Furthermore, recall bias cannot be excluded as it can lead to under or overestimation of DP intakes despite meticulous data collection. Regarding the assessment of frailty, we complemented slowness and handgrip strength with the Rosow-Breslau scale and the chair stand test, respectively, to minimize the loss of participants due to missing data. Indeed, the Rosow-Breslau scale has been shown to be strongly associated with walking [38] and the chair stand test was shown to be a good proxy for handgrip strength $[39,53]$. Furthermore, we were not able to check frailty incidence over 10-years at different waves of follow-up because the frailty phenotype could not be calculated at each time interval. Nevertheless, this limit was toned down when using the FRAIL scale, which identified a lower number of frail participants, but provided similar results on the DP-frailty associations in both the cross-sectional and prospective analyses. Despite these similarities, we speculate that the imbalance between frail and non-frail groups might have led to underpowered comparisons, hindering the observation of real differences if any. In addition, a selection bias cannot be dismissed, since not included participants (cross-sectional sample) were older, had lower educational levels and cognitive performance, had more frequent depressive symptoms, multi-morbidities, polypharmacy, and worse diet scores than included participants (data not shown). Finally, although we adjusted for several major covariates, some residual confounding factors cannot be dismissed. In fact, we acknowledge that the collected dietary data dates back to 2000, which is old, and this might affect the relevance of our results. Nevertheless, the French RDA applied to the year of data collection (2000-2001) is still applied till now and it has been previously reported that intakes of major food groups appeared to be relatively stable during follow-up in 3C Bordeaux [54].

Despite these limitations, the current study has several strengths. First, we focused our analyses on a large sample of French elderly consumers, known to exhibit distinctive DP consumption, notably cheese [31], within a population-based setting while adjusting for major confounders (note, less than $0.1 \%$ of $3 \mathrm{C}$-Bordeaux participants were consumers of food supplements at baseline, which precluded using this data as a confounder). Second, survival analyses were performed to check if there is any competitive risk with death (data not shown). We observed that DP exposures were not significantly associated with mortality, eliminating the selection bias leading to survival effect often faced in prospective studies involving older adults. Moreover, we confirmed our main results using a different scale to assess frailty and when keeping participants who exhibited dependency in both cross-sectional and prospective studied samples, which allowed us to further decrease the selection bias (i.e., frailty being considered as a pre-dependency stage and risk factor for disability $[35,55])$.

In conclusion, we did not observe any association between DP consumption, whatever the sub-types, and frailty prevalence or incidence among this sample of French older adults. Studies on this topic are scarce and future studies are still needed while taking into consideration the identified limitations, such as the potential benefits/risks ratio of DP nutrient contents. In the meantime, and beyond frailty, older adults are encouraged to follow French nutritional recommendations for DP consumption (2 to 3 times/d) as their benefits on the general well-being of older adults to prevent osteoporosis and malnutrition 
are well established, and recent large-scale settings have also suggested their protective ability to prevent chronic diseases and mortality.

Author Contributions: Conceptualization, C.F.; Formal analysis, H.P.; Methodology, B.R., H.P. and C.F.; Project administration, C.H. and C.F.; Resources, C.S. and C.F.; Supervision, C.F.; Validation, C.F.; Writing—original draft, B.R. and H.P.; Writing—review \& editing, V.C., C.H., C.S. and C.F. All authors have read and agreed to the published version of the manuscript.

Funding: This research received no external funding.

Institutional Review Board Statement: The 3C study was approved by the Consultative Committee for the Protection of Persons participating in Biomedical Research at Kremlin-Bicêtre.

Informed Consent Statement: Informed consent was obtained from all subjects involved in the study.

Data Availability Statement: Data described in the manuscript, code book and analytic code will be made available upon request: http:/ /www.three-city-study.com/ancillary-studies.php (accessed on 5 April 2021).

Acknowledgments: The Three-City Study is conducted under a partnership agreement between the Institut National de la Santé et de la Recherche Médicale (INSERM), Victor Segalen-Bordeaux2 University and the Sanofi-Synthélabo company. The Fondation pour la Recherche Médicale funded the preparation and beginning of the study. The 3C-Study is also sponsored by the Caisse Nationale Maladie des Travailleurs Salariés, Direction Générale de la Santé, Conseils Régionaux of Aquitaine and Bourgogne, Fondation de France, Ministry of Research-INSERM Program Cohortes et collections de données biologiques, the Fondation Plan Alzheimer (FCS 2009-2012), the Caisse Nationale pour la Solidarité et l'Autonomie (CNSA) and the "Programme Longévité et vieillissement", COGICARE 07-LVIE 003 01, the FRAILOMIC Initiative (FP7-HEALTH-2012-Proposal No. 305483-2).

Conflicts of Interest: The authors declare no conflict of interest regarding the current work.

\section{References}

1. Department of Economic and Social Affairs, U.N. World Population Prospects 2019: Highlights. Available online: https: / / population.un.org/wpp/Publications/Files/WPP2019_10KeyFindings.pdf (accessed on 5 April 2021).

2. World Health Organization. "Ageing Well” Must Be a Global Priority. Available online: https://http://www.who.int/news/ item/06-11-2014--ageing-well-must-be-a-global-priority (accessed on 5 April 2021).

3. Tabue-Teguo, M.; Grasset, L.; Avila-Funes, J.A.; Genuer, R.; Proust-Lima, C.; Péres, K.; Féart, C.; Amieva, H.; Harmand, M.G.; Helmer, C.; et al. Prevalence and Co-Occurrence of Geriatric Syndromes in People Aged 75 Years and Older in France: Results From the Bordeaux Three-city Study. J. Gerontol. Ser. A Biol. Sci. Med Sci. 2017, 73, 109-116. [CrossRef]

4. Fried, L.P.; Tangen, C.M.; Walston, J.; Newman, A.B.; Hirsch, C.; Gottdiener, J.; Seeman, T.; Tracy, R.; Kop, W.J.; Burke, G.; et al. Frailty in older adults: Evidence for a phenotype. J. Gerontol. Ser. A Biol. Sci. Med Sci. 2001, 56, M146-M156. [CrossRef] [PubMed]

5. Mareschal, J.; Genton, L.; Collet, T.H.; Graf, C. Nutritional Intervention to Prevent the Functional Decline in Community-Dwelling Older Adults: A Systematic Review. Nutrients 2020, 12, 2820. [CrossRef]

6. Feart, C. Nutrition and frailty: Current knowledge. Prog. Neuro Psychopharmacol. Biol. Psychiatry 2019, 95, 109703. [CrossRef]

7. Sandoval-Insausti, H.; Pérez-Tasigchana, R.F.; López-García, E.; García-Esquinas, E.; Rodríguez-Artalejo, F.; Guallar-Castillón, P. Macronutrients Intake and Incident Frailty in Older Adults: A Prospective Cohort Study. J. Gerontol. Ser. A Biol. Sci. Med Sci. 2016, 71, 1329-1334. [CrossRef]

8. Shikany, J.M.; Barrett-Connor, E.; Ensrud, K.E.; Cawthon, P.M.; Lewis, C.E.; Dam, T.T.; Shannon, J.; Redden, D.T. Macronutrients, diet quality, and frailty in older men. J. Gerontol. Ser. A Biol. Sci. Med Sci. 2014, 69, 695-701. [CrossRef]

9. García-Esquinas, E.; Rahi, B.; Peres, K.; Colpo, M.; Dartigues, J.F.; Bandinelli, S.; Feart, C.; Rodríguez-Artalejo, F. Consumption of fruit and vegetables and risk of frailty: A dose-response analysis of 3 prospective cohorts of community-dwelling older adults. Am. J. Clin. Nutr. 2016, 104, 132-142. [CrossRef]

10. Fung, T.T.; Struijk, E.A.; Rodriguez-Artalejo, F.; Willett, W.C.; Lopez-Garcia, E. Fruit and vegetable intake and risk of frailty in women 60 years old or older. Am. J. Clin. Nutr. 2020, 112, 1540-1546. [CrossRef] [PubMed]

11. Del Brutto, O.H.; Mera, R.M.; Ha, J.E.; Gillman, J.; Zambrano, M.; Sedler, M.J. Dietary Oily Fish Intake and Frailty. A PopulationBased Study in Frequent Fish Consumers Living in Rural Coastal Ecuador (the Atahualpa Project). J. Nutr. Gerontol. Geriatr. 2020, 39, 88-97. [CrossRef]

12. Rashidi Pour Fard, N.; Amirabdollahian, F.; Haghighatdoost, F. Dietary patterns and frailty: A systematic review and metaanalysis. Nutr. Rev. 2019, 77, 498-513. [CrossRef]

13. Feng, Z.; Lugtenberg, M.; Franse, C.; Fang, X.; Hu, S.; Jin, C.; Raat, H. Risk factors and protective factors associated with incident or increase of frailty among community-dwelling older adults: A systematic review of longitudinal studies. PLoS ONE 2017, 12, e0178383. [CrossRef] 
14. Leon-Munoz, L.M.; Garcia-Esquinas, E.; Lopez-Garcia, E.; Banegas, J.R.; Rodriguez-Artalejo, F. Major dietary patterns and risk of frailty in older adults: A prospective cohort study. BMC Med. 2015, 13, 11. [CrossRef]

15. Struijk, E.A.; Hagan, K.A.; Fung, T.T.; Hu, F.B.; Rodríguez-Artalejo, F.; Lopez-Garcia, E. Diet quality and risk of frailty among older women in the Nurses' Health Study. Am. J. Clin. Nutr. 2020, 111, 877-883. [CrossRef] [PubMed]

16. Lorenzo-López, L.; Maseda, A.; de Labra, C.; Regueiro-Folgueira, L.; Rodríguez-Villamil, J.L.; Millán-Calenti, J.C. Nutritional determinants of frailty in older adults: A systematic review. BMC Geriatr. 2017, 17, 108. [CrossRef]

17. Rahi, B.; Ajana, S.; Tabue-Teguo, M.; Dartigues, J.F.; Peres, K.; Feart, C. High adherence to a Mediterranean diet and lower risk of frailty among French older adults community-dwellers: Results from the Three-City-Bordeaux Study. Clin. Nutr. 2018, 37, 1293-1298. [CrossRef]

18. Rahi, B.; Colombet, Z.; Gonzalez-Colaco Harmand, M.; Dartigues, J.F.; Boirie, Y.; Letenneur, L.; Feart, C. Higher Protein but Not Energy Intake Is Associated With a Lower Prevalence of Frailty Among Community-Dwelling Older Adults in the French Three-City Cohort. J. Am. Med Dir. Assoc. 2016, 17, 672.e7-672.e11. [CrossRef]

19. Coelho-Júnior, H.J.; Rodrigues, B.; Uchida, M.; Marzetti, E. Low Protein Intake Is Associated with Frailty in Older Adults: A Systematic Review and Meta-Analysis of Observational Studies. Nutrients 2018, 10, 1334. [CrossRef] [PubMed]

20. Mendonça, N.; Kingston, A.; Granic, A.; Jagger, C. Protein intake and transitions between frailty states and to death in very old adults: The Newcastle 85+ study. Age Ageing 2019, 49, 32-38. [CrossRef] [PubMed]

21. Isanejad, M.; Sirola, J.; Rikkonen, T.; Mursu, J.; Kröger, H.; Qazi, S.L.; Tuppurainen, M.; Erkkilä, A.T. Higher protein intake is associated with a lower likelihood of frailty among older women, Kuopio OSTPRE-Fracture Prevention Study. Eur. J. Nutr. 2020, 59, 1181-1189. [CrossRef] [PubMed]

22. Drouin-Chartier, J.P.; Li, Y.; Ardisson Korat, A.V.; Ding, M.; Lamarche, B.; Manson, J.E.; Rimm, E.B.; Willett, W.C.; Hu, F.B. Changes in dairy product consumption and risk of type 2 diabetes: Results from 3 large prospective cohorts of US men and women. Am. J. Clin. Nutr. 2019, 110, 1201-1212. [CrossRef]

23. Mitri, J.; Mohd Yusof, B.N.; Maryniuk, M.; Schrager, C.; Hamdy, O.; Salsberg, V. Dairy intake and type 2 diabetes risk factors: A narrative review. Diabetes Metab. Syndr. 2019, 13, 2879-2887. [CrossRef] [PubMed]

24. Dehghan, M.; Mente, A.; Rangarajan, S.; Sheridan, P.; Mohan, V.; Iqbal, R.; Gupta, R.; Lear, S.; Wentzel-Viljoen, E.; Avezum, A.; et al. Association of dairy intake with cardiovascular disease and mortality in 21 countries from five continents (PURE): A prospective cohort study. Lancet 2018, 392, 2288-2297. [CrossRef]

25. Ding, M.; Li, J.; Qi, L.; Ellervik, C.; Zhang, X.; Manson, J.E.; Stampfer, M.; Chavarro, J.E.; Rexrode, K.M.; Kraft, P.; et al. Associations of dairy intake with risk of mortality in women and men: Three prospective cohort studies. BMJ Clin. Res. Ed. 2019, 367, 16204. [CrossRef]

26. Guo, J.; Astrup, A.; Lovegrove, J.A.; Gijsbers, L.; Givens, D.I.; Soedamah-Muthu, S.S. Milk and dairy consumption and risk of cardiovascular diseases and all-cause mortality: Dose-response meta-analysis of prospective cohort studies. Eur. J. Epidemiol. 2017, 32, 269-287. [CrossRef] [PubMed]

27. Cuesta-Triana, F.; Verdejo-Bravo, C.; Fernández-Pérez, C.; Martín-Sánchez, F.J. Effect of Milk and Other Dairy Products on the Risk of Frailty, Sarcopenia, and Cognitive Performance Decline in the Elderly: A Systematic Review. Adv. Nutr. 2019, 10, S105-S119. [CrossRef] [PubMed]

28. Hanach, N.I.; McCullough, F.; Avery, A. The Impact of Dairy Protein Intake on Muscle Mass, Muscle Strength, and Physical Performance in Middle-Aged to Older Adults with or without Existing Sarcopenia: A Systematic Review and Meta-Analysis. Adv. Nutr. 2019, 10, 59-69. [CrossRef]

29. Lana, A.; Rodriguez-Artalejo, F.; Lopez-Garcia, E. Dairy Consumption and Risk of Frailty in Older Adults: A Prospective Cohort Study. J. Am. Geriatr. Soc. 2015, 63, 1852-1860. [CrossRef]

30. Ribeiro, I.; Gomes, M.; Figueiredo, D.; Lourenço, J.; Paúl, C.; Costa, E. Dairy Product Intake in Older Adults across Europe Based On the SHARE Database. J. Nutr. Gerontol. Geriatr. 2019, 38, 297-306. [CrossRef]

31. Pellay, H.; Marmonier, C.; Samieri, C.; Feart, C. Socio-Demographic Characteristics, Dietary, and Nutritional Intakes of French Elderly Community Dwellers According to Their Dairy Product Consumption: Data from the Three-City Cohort. Nutrients 2020, 12, 3418. [CrossRef]

32. 3C Study Group. Vascular factors and risk of dementia: Design of the Three-City Study and baseline characteristics of the study population. Neuroepidemiology 2003, 22, 316-325. [CrossRef]

33. Samieri, C.; Jutand, M.A.; Féart, C.; Capuron, L.; Letenneur, L.; Barberger-Gateau, P. Dietary patterns derived by hybrid clustering method in older people: Association with cognition, mood, and self-rated health. J. Am. Diet. Assoc. 2008, 108, $1461-1471$. [CrossRef]

34. Simermann, J.; Barberger-Gateau, P.; Berr, C. Validation of a Food Frequency Questionnaire in older population. In Proceedings of the 25th annual congress of SFNEP: Clinical Nutrition and Metabolism, Montpellier, France, 28-30 November 2007.

35. Dent, E.; Morley, J.E.; Cruz-Jentoft, A.J.; Woodhouse, L.; Rodríguez-Mañas, L.; Fried, L.P.; Woo, J.; Aprahamian, I.; Sanford, A.; Lundy, J.; et al. Physical Frailty: ICFSR International Clinical Practice Guidelines for Identification and Management. J. Nutr. Health Aging 2019, 23, 771-787. [CrossRef]

36. Radloff, L.S. The CES-D scale a self-report depression scale for research in the general population. Appl. Psychol. Meas. 1977, 1, 385-401. [CrossRef]

37. Rosow, I.; Breslau, N. A Guttman health scale for the aged. J. Gerontol. 1966, 21, 556-559. [CrossRef] 
38. Alexander, N.B.; Guire, K.E.; Thelen, D.G.; Ashton-Miller, J.A.; Schultz, A.B.; Grunawalt, J.C.; Giordani, B. Self-reported walking ability predicts functional mobility performance in frail older adults. J. Am. Geriatr. Soc. 2000, 48, 1408-1413. [CrossRef] [PubMed]

39. Avila-Funes, J.A.; Helmer, C.; Amieva, H.; Barberger-Gateau, P.; Le Goff, M.; Ritchie, K.; Portet, F.; Carriere, I.; Tavernier, B.; Gutierrez-Robledo, L.M.; et al. Frailty among community-dwelling elderly people in France: The three-city study. J. Gerontol. Ser. A Biol. Sci. Med Sci. 2008, 63, 1089-1096. [CrossRef] [PubMed]

40. Morley, J.E.; Malmstrom, T.K.; Miller, D.K. A simple frailty questionnaire (FRAIL) predicts outcomes in middle aged African Americans. J. Nutr. Health Aging 2012, 16, 601-608. [CrossRef]

41. Katz, S.; Ford, A.B.; Moskowitz, R.W.; Jackson, B.A.; Jaffe, M.W. Studies of Illness in the Aged. The Index of ADL: A Standardized Measure of Biological and Psychosocial Function. JAMA J. Am. Med Assoc. 1963, 185, 914-919. [CrossRef] [PubMed]

42. Folstein, M.F.; Folstein, S.E.; McHugh, P.R. "Mini-mental state". A practical method for grading the cognitive state of patients for the clinician. J. Psychiatr. Res. 1975, 12, 189-198. [CrossRef]

43. Feart, C.; Jutand, M.A.; Larrieu, S.; Letenneur, L.; Delcourt, C.; Combe, N.; Barberger-Gateau, P. Energy, macronutrient and fatty acid intake of French elderly community dwellers and association with socio-demographic characteristics: Data from the Bordeaux sample of the Three-City Study. Br. J. Nutr. 2007, 98, 1046-1057. [CrossRef]

44. Gil, Á.; Ortega, R.M. Introduction and Executive Summary of the Supplement, Role of Milk and Dairy Products in Health and Prevention of Noncommunicable Chronic Diseases: A Series of Systematic Reviews. Adv. Nutr. 2019, 10, S67-S73. [CrossRef] [PubMed]

45. Radavelli-Bagatini, S.; Zhu, K.; Lewis, J.R.; Dhaliwal, S.S.; Prince, R.L. Association of dairy intake with body composition and physical function in older community-dwelling women. J. Acad. Nutr. Diet. 2013, 113, 1669-1674. [CrossRef] [PubMed]

46. Tanaka, T.; Talegawkar, S.A.; Jin, Y.; Bandinelli, S.; Ferrucci, L. Association of Adherence to the Mediterranean-Style Diet with Lower Frailty Index in Older Adults. Nutrients 2021, 13, 1129. [CrossRef] [PubMed]

47. Agence Nationale de Sécurité Sanitaire de L'alimentation, d.l.e.e.d.t.A. INCA 3 : Evolution des Habitudes et Modes de Consommation, de Nouveaux Enjeux en Matière de Sécurité Sanitaire et de Nutrition. Available online: https://http:/ / www.anses.fr/fr/ content/inca-3-evolution-des-habitudes-et-modes-de-consommation-de-nouveaux-enjeux-en-mati\%C3\%A8re-de (accessed on 5 April 2021).

48. $\mathrm{Hu}, \mathrm{F}$. Dietary pattern analysis: A new direction in nutritional epidemiology. Curr. Opin. Lipidol. 2002, 13, 3-9. [CrossRef]

49. Laclaustra, M.; Rodriguez-Artalejo, F.; Guallar-Castillon, P.; Banegas, J.R.; Graciani, A.; Garcia-Esquinas, E.; Ordovas, J.; Lopez-Garcia, E. Prospective association between added sugars and frailty in older adults. Am. J. Clin. Nutr. 2018, 107, 772-779. [CrossRef] [PubMed]

50. Jayanama, K.; Theou, O.; Godin, J.; Cahill, L.; Rockwood, K. Association of fatty acid consumption with frailty and mortality among middle-aged and older adults. Nutrition 2020, 70, 110610. [CrossRef] [PubMed]

51. Artaza-Artabe, I.; Sáez-López, P.; Sánchez-Hernández, N.; Fernández-Gutierrez, N.; Malafarina, V. The relationship between nutrition and frailty: Effects of protein intake, nutritional supplementation, vitamin D and exercise on muscle metabolism in the elderly. A systematic review. Maturitas 2016, 93, 89-99. [CrossRef]

52. Bruyère, O.; Cavalier, E.; Buckinx, F.; Reginster, J.Y. Relevance of vitamin D in the pathogenesis and therapy of frailty. Curr. Opin. Clin. Nutr. Metab. Care 2017, 20, 26-29. [CrossRef]

53. Rantanen, T.; Era, P.; Kauppinen, M.; Heikkinen, E. Maximal isometric muscle strength and socioeconomic status, health, and physical activity in 75-year-old persons. J. Aging Phys. Act. 1994, 2, 206-220. [CrossRef]

54. Pelletier, A.; Barul, C.; Feart, C.; Helmer, C.; Bernard, C.; Periot, O.; Dilharreguy, B.; Dartigues, J.F.; Allard, M.; Barberger-Gateau, P.; et al. Mediterranean diet and preserved brain structural connectivity in older subjects. Alzheimer Dement. J. Alzheimer Assoc. 2015, 11, 1023-1031. [CrossRef]

55. Liu, H.X.; Ding, G.; Yu, W.J.; Liu, T.F.; Yan, A.Y.; Chen, H.Y.; Zhang, A.H. Association between frailty and incident risk of disability in community-dwelling elder people: Evidence from a meta-analysis. Public Health 2019, 175, 90-100. [CrossRef] [PubMed] 